\title{
Dark-Bright Solitons Conversion System for Secured and Long Distance Optical Communication
}

\author{
I. S. Amiri ${ }^{1}$, S. Babakhani ${ }^{2}$, G. R. Vahedi ${ }^{2}$, J. Ali ${ }^{1}$, P. P. Yupapin ${ }^{3}$ \\ ${ }^{1}$ Institute of Advanced Photonics Science, Nanotechnology Research Alliance, Universiti Teknologi Malaysia \\ (UTM), 81310 Johor Bahru, Malaysia \\ ${ }^{2}$ Faculty of Science, Department of Physics, Universiti Putra Malaysia, 43400 UPM Serdang \\ ${ }^{3}$ Advanced Research Center for Photonics, Faculty of Science King Mongkut's Institute of Technology \\ Ladkrabang Bangkok 10520, Thailand
}

\begin{abstract}
We suggest a new purpose of a security scheme by employing the nonlinear behaviors of temporal dark and bright solitons amongst a micro-ring resonator system for signal security application. The chaotic signal is generated, where the required bright soliton pulse can be recovered and discovered by an add/drop filtering device. By using the reserve ring parameters, simulation results obtained have demonstrated that the soliton conversion can be performed. In application, the chaotic signal is generated and formed by the dark soliton inside a nonlinear micro-ring device. The different temporal soliton response time can be seen, the response times of 169 and 84 ns are mentioned for temporal dark and bright solitons, respectively, which can also be used to figure the security key. The technique of optical conversion can be use to improve the optical communication network systems.
\end{abstract}

Keyword: microring resonator, nonlinear medium, chaotic signals, add/drop filter, dark-bright conversion

\section{INTRODUCTION}

Dark and bright soliton behaviors have been widely investigated in different forms [1]. Dark soliton is one of the soliton properties, whereas the soliton amplitude disappears or minimized throughout the propagation in media, thus, the dark soliton detection is difficult [2]. The investigation of dark soliton behaviors has been described, where one point of them has shown the interesting results, where the dark soliton can be stabilized and converted into bright soliton and eventually observed $[3,4]$. This means that we can employ the dark soliton penalty because of the low level of the peak power to be the benefit, where the predicting idea is that a dark soliton can perform the communication transmission carrier where the recovery can be retrieved by the darkbright soliton conversion [5]. A soliton pulse can be localized among a waveguide consists of micro and nanoring resonator, hence the soliton pulse can be stored within the nano-waveguide [6]. We have shown that the dark soliton can be input and chopped to the noisy signals for security use within the nonlinear ring resonator system [7]. The required users can retrieve the original signal through an add/drop filter, where they can select to retrieve in either bright or dark soliton pulses $[8,9]$. The filtering feature of the optical signal is presented within an add/drop filter, where the suitable parameters can be operated to obtain the required output energy [10].

\section{THEORY}

The dark soliton pulse, which is introduced into the multi-stage micro-ring resonators as shown in figure1, the input optical field $\left(E_{i n}\right)$ of the dark soliton pulse input is given by $[11,12]$

$$
E_{i n}=A \tanh \left[\frac{T}{T_{0}}\right] \exp \left[\left(\frac{z}{2 L_{D}}\right)-i \omega_{0} t\right]
$$

where $A$ and $z$ are the optical field amplitude and propagation distance, respectively [13,14]. $T$ is a soliton pulse propagation time in a frame moving at the group velocity $[15,16], T=t-\beta_{1} \times z$, where $\beta_{1}$ and $\beta_{2}$ are the coefficients of the linear and second-order terms of Taylor expansion of the propagation constant [17]. $L_{D}=T_{0}^{2} /\left|\beta_{2}\right|$ is the dispersion length of the soliton pulse [18]. $T_{0}$ in equation (1) is a soliton pulse propagation time at initial input [19]. Where $t$ is the soliton phase shift time, and the frequency shift of the soliton is $\omega_{0}[20,21]$. 


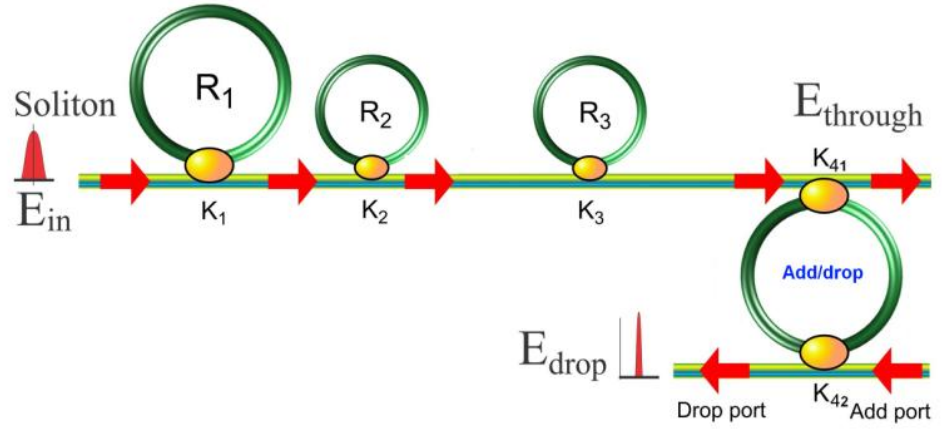

Fig. 1: Dark-bright soliton conversion system, where $R_{\mathrm{s}}$ : ring radii, $\kappa_{\mathrm{s}}$ : coupling coefficients, $\kappa_{41}$ and $\kappa_{42}$ are the add/drop coupling coefficients

The refractive index $(n)$ of light within the medium is given by [22]

$$
n=n_{0}+n_{2} I=n_{0}+\left(\frac{n_{2}}{A_{e f f}}\right) P,
$$

where $n_{0}$ and $n_{2}$ are the linear and nonlinear refractive indexes, respectively [23]. $I$ and $P$ are the optical intensity and optical power, respectively [24]. The effective mode core area of the device is given by $A_{\text {eff }}$ and ranges from 0.50 to $0.10 \mu \mathrm{m}^{2}[25,26]$. The normalized output of the light field in each roundtrip is given by [27]

$$
\left|\frac{E_{\text {out }}(t)}{E_{\text {in }}(t)}\right|^{2}=(1-\gamma)\left[1-\frac{\left(1-(1-\gamma) x^{2}\right) \kappa}{(1-x \sqrt{1-\gamma} \sqrt{1-\kappa})^{2}+4 x \sqrt{1-\gamma} \sqrt{1-\kappa} \sin ^{2}\left(\frac{\phi}{2}\right)}\right]
$$

$\kappa$ is the coupling coefficient, and $x=\exp (-\alpha L / 2)$ represents a roundtrip loss coefficient, $\varphi_{0}=k L n_{0}$ and $\varphi_{N L}=k L n_{2}\left|E_{i n}\right|^{2}$ are the linear and nonlinear phase shifts, $k=2 \pi / \lambda$ is the wave propagation number in a vacuum [28]. Where $L$ and $\alpha$ are a waveguide length and linear absorption coefficient, respectively [29, 30]. To retrieve the signals from the chaotic noise, we propose to use the add/drop device with the appropriate parameters [31, 32]. Optical circuits of ring resonator add/drop filters for the throughput and drop port can be given by $[33,34$, 35]

$$
\left|\frac{E_{t}}{E_{\text {in }}}\right|^{2}=\frac{\left(1-\kappa_{41}\right)-2 \sqrt{1-\kappa_{41}} \cdot \sqrt{1-\kappa_{42}} e^{-\frac{\alpha}{2} L} \cos \left(k_{n} L\right)+\left(1-\kappa_{42}\right) e^{-\alpha L}}{1+\left(1-\kappa_{41}\right)\left(1-\kappa_{42}\right) e^{-\alpha L}-2 \sqrt{1-\kappa_{41}} \cdot \sqrt{1-\kappa_{42}} e^{-\frac{\alpha}{2} L} \cos \left(k_{n} L\right)}
$$

and

$$
\left|\frac{E_{d}}{E_{\text {in }}}\right|^{2}=\frac{\kappa_{41} \kappa_{42} e^{-\frac{\alpha}{2} L}}{1+\left(1-\kappa_{41}\right)\left(1-\kappa_{42}\right) e^{-\alpha L}-2 \sqrt{1-\kappa_{41}} \cdot \sqrt{1-\kappa_{42}} e^{-\frac{\alpha}{2} L} \cos \left(k_{n} L\right)}
$$

where $E_{t}$ and $E_{d}$ represents the optical fields of the throughput and drop ports, respectively [36]. $\beta=k n_{\text {eff }}$ is the propagation constant, $n_{\text {eff }}$ is the effective refractive index of the waveguide and the circumference of the ring is $L=2 \pi R$, here $R$ is the radius of the ring [37, 38]. $\kappa_{41}$ and $\kappa_{42}$ are coupling coefficient of add/drop filters, $k_{n}=2 \pi / \lambda$ is the wave propagation number for in a vacuum, and where the waveguide (ring resonator) loss is $\alpha=0.5 \mathrm{~dB} \mathrm{~mm}^{-1}$ $[39,40]$. The fractional coupler intensity loss is $\gamma=0.1[41,42]$. In the case of add/drop device, the nonlinear refractive index is neglected $[43,44]$.

\section{Result And Discussion}

Dark soliton pulse with $50 \mathrm{~ns}$ pulse width, the maximum power of $1.0 \mathrm{~W}$ is input into the dark-bright solitons conversion system as demonstrated in figure 1. The suitable ring parameters are applied, for example, ring radii $R_{1}=10.0 \mu \mathrm{m}, R_{2}=7.0 \mu \mathrm{m}$ and $R_{3}=5.0 \mu \mathrm{m}$. The system are prepared to $\lambda_{0}=1.55 \mu \mathrm{m}, n_{0}=3.34$ (InGaAsP/InP), $A_{\text {eff }}=0.50,0.25$ and $0.10 \mu \mathrm{m}^{2}$ for a micro and nanoring resonators, respectively, $\alpha=0.5 \mathrm{~dB} \mathrm{~mm}^{-1}$, $\gamma=0.1$. The coupling coefficients (kappa, $\kappa$ ) of the micro-ring resonator are ranged from 0.05 to 0.90 . The nonlinear refractive index is $n_{2}=1.2 \times 10^{-17} \mathrm{~m}^{2} / \mathrm{W}$. The input dark soliton pulse is chopped (sliced) into the 
Dark-Bright Solitons Conversion System for Secured and Long Distance Optical Communication smaller signals as shown in figure 2(a). Figure 2(b) and (c) are the output signals of the filtering signals within the rings $R_{2}$ and $R_{3}$. The soliton signals in $R_{3}$ is inserted into the add/drop filter, where the dark-bright solitons conversion can be performed by using equations (4) and (5).
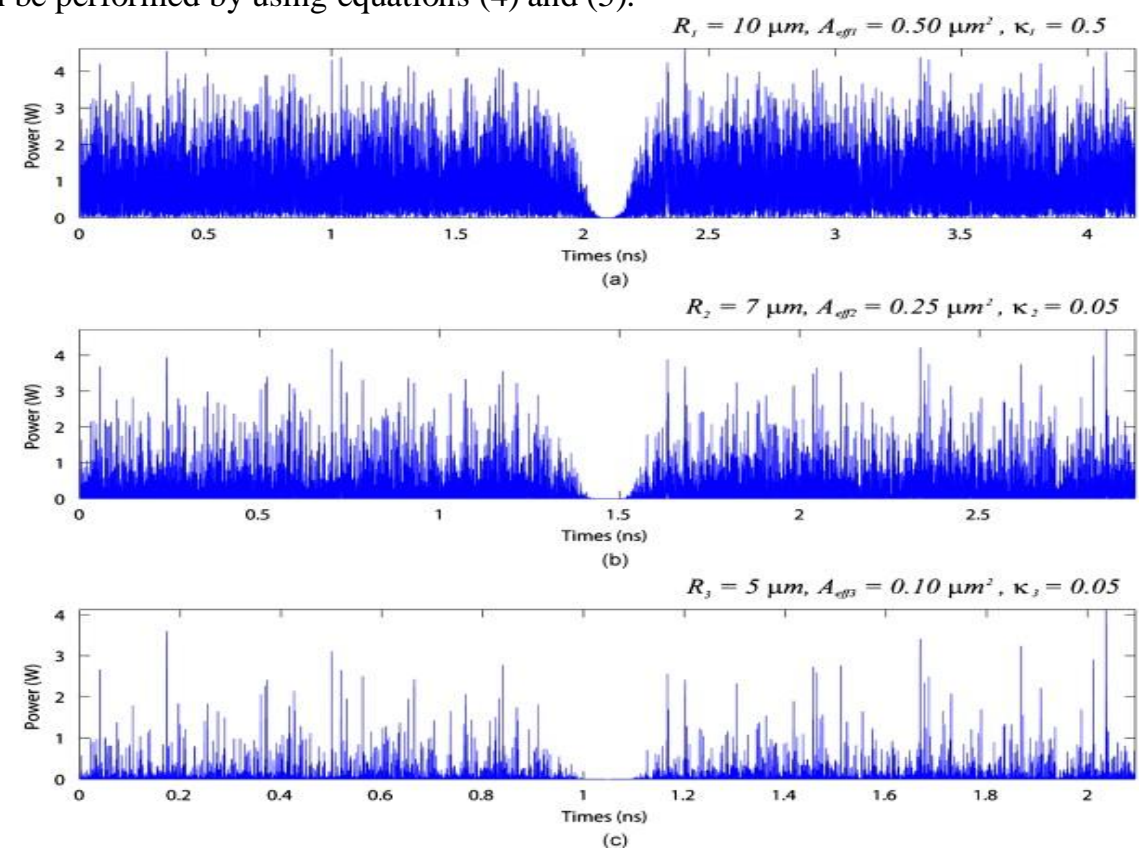

Fig. 2: Simulation results of the soliton signals within the ring resonator system, where (a): $R_{1}$ output power, (b): $R_{2}$ output power and (c): $R_{3}$ output power.

Dark soliton pulse is input into a micro and nanoring resonator systems as shown in figure 3 and figure 4. The add/drop filter is connected to two couplers where the ring radius $\left(R_{d}\right)$ is $10 \mu \mathrm{m}$ and the coupling constants $\left(\kappa_{11}\right.$ and $\left.\kappa_{12}\right)$ are the same values $(0.50)$. When the add/drop filter is connected to the third ring $\left(R_{3}\right)$, the dark-bright solitons conversion are seen. The bright soliton and dark solitons are observed by the through and drop ports as demonstrated in figure 1 , respectively.

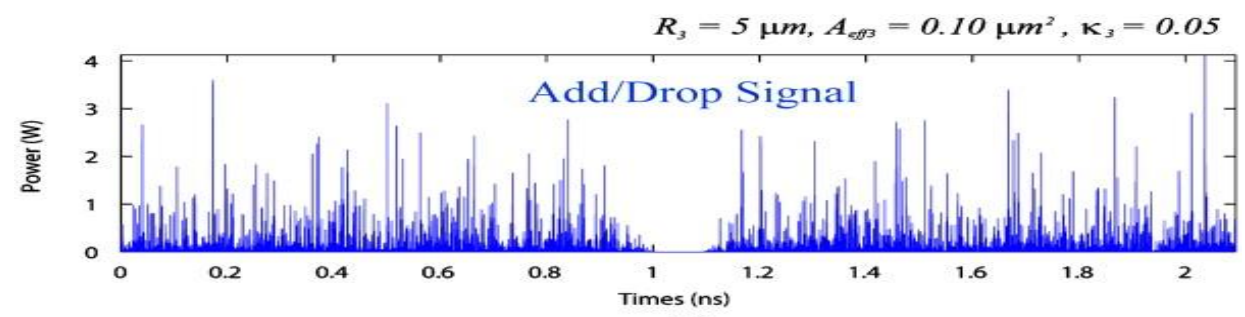

(a)

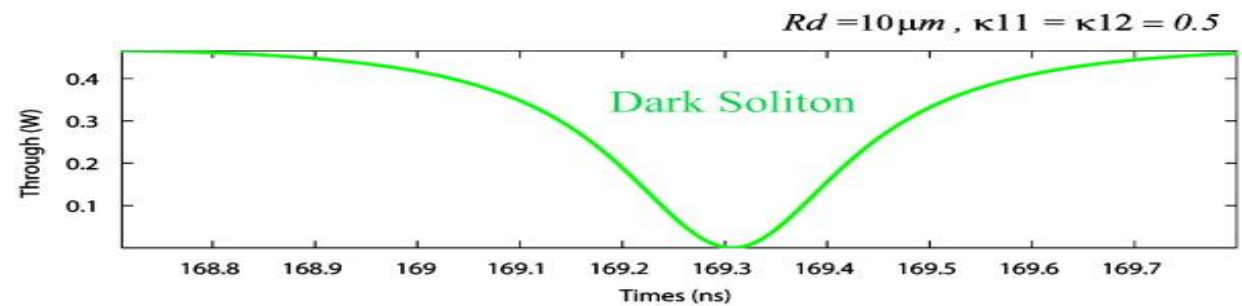

(b)

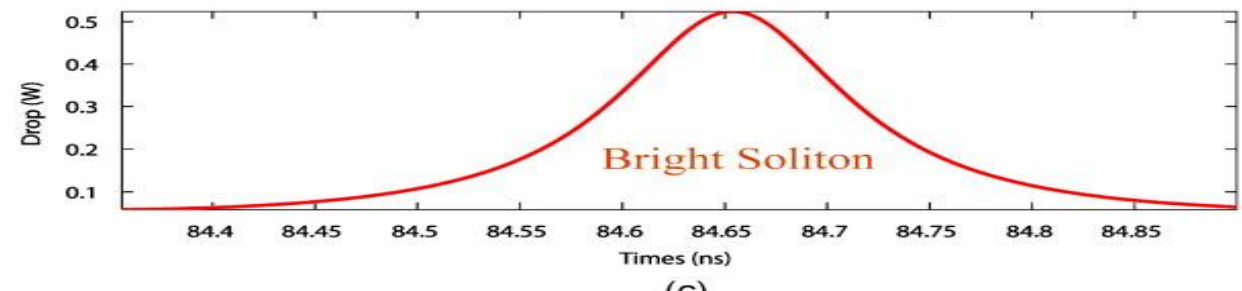

(c)

Fig. 3: Results of the optical solitons, where (a) the signals in $R_{3}$, (b) a dark soliton and (c) a bright soliton. the input dark soliton power is $1 \mathrm{~W}, \kappa_{1}=0.5, R_{d}=10 \mathrm{~mm}$. 


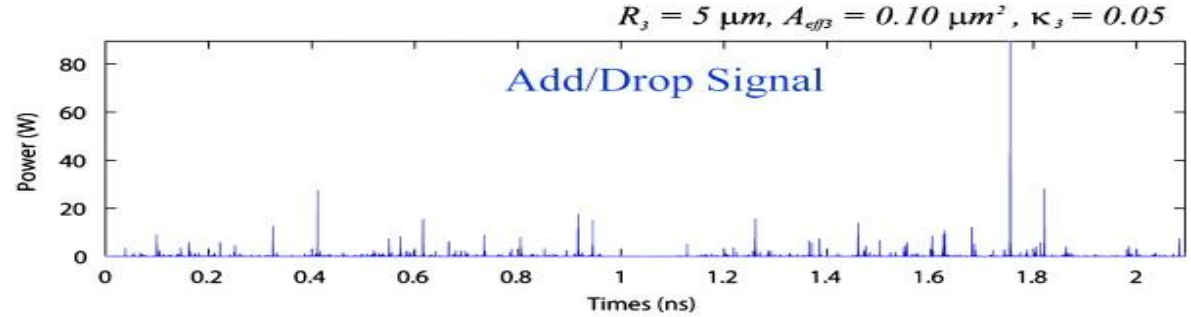

(a)

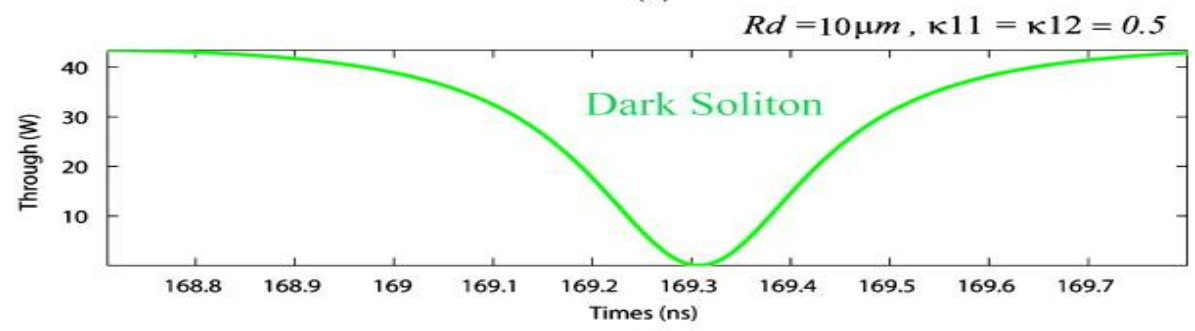

(b)



(c)

Fig. 4: Results of the optical solitons, where (a): the signals in $R_{3}$, (b): a dark soliton and (c): a bright soliton, where input dark soliton power is $1 \mathrm{~W}, \kappa_{1}=0.9, R_{d}=10 \mathrm{~mm}$.

The system of conversion optical soliton pulses can be used in optical communication network where the security can be performed by use of dark soliton. The bright soliton is used for long distance optical communication where the optical conversion system allows the different users to receive suitable type of optical solitons depending of the required application. Schematic of an optical communication network system is shown in figure 5 where the system of conversion can be seen along the link.

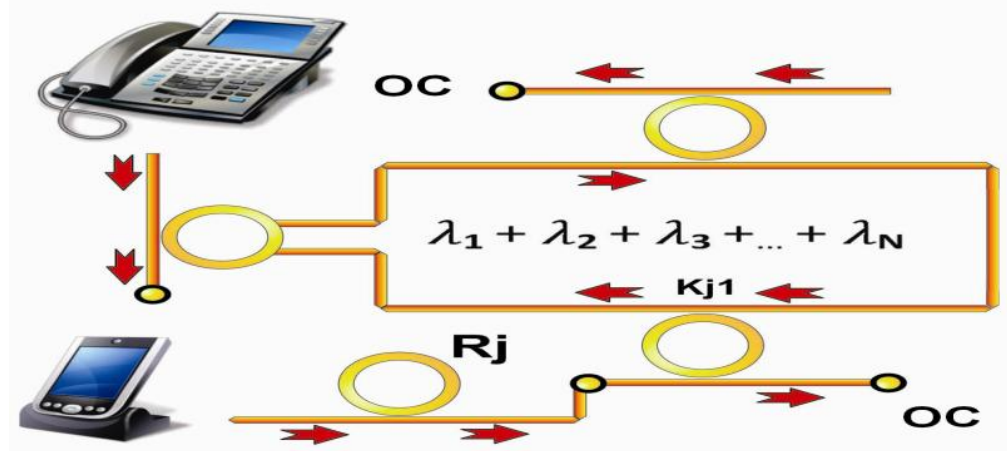

Fig. 5: Schematic of an optical communication network system where $R_{j}$ : ring's radius, $\lambda_{\mathrm{i}}$ : transmitting wavelength, $\kappa_{\mathrm{j}}$ : coupling coefficient, OC: optical conversion system

\section{Conclusion}

In conclusion, dark soliton in the optical microring resonator can be converted to be a bright soliton incorporating the add/drop system. By using the reasonable dark soliton input power, the output bright soliton power obtained can be used to perform the common soliton for long-distance link, for instance, the output power of bright solitons of 0.5 and $40 \mathrm{~W}$ are obtained. The advantage is that the detection of the dark soliton along the through port is difficult, while the detection of the bright one can be performed by the standard form. This means the use of dark soliton to form the signal security or communication security is plausible, which is also available for network security application.

\section{Acknowledgements}

We would like to thank the Institute of Advanced Photonics Science, Nanotechnology Research Alliance, Universiti Teknologi Malaysia (UTM) and IDF financial support from UTM. 


\section{References}

[1] A. Afroozeh, I.S. Amiri, M. Kouhnavard, M. Jalil, J. Ali, and P. Yupapin. Optical dark and bright soliton generation and amplification. Enabling Science and Nanotechnology (ESciNano) Conference 2010. Malaysia: IEEE Explore.

[2] I.S. Amiri, A. Afroozeh, I. Nawi, M. Jalil, A. Mohamad, J. Ali, and P. Yupapin, Dark Soliton Array for Communication Security, Procedia Engineering, 8, 2011, 417-422.

[3] M. Bahadoran, I.S. Amiri, A. Afroozeh, J. Ali, and P.P. Yupapin, Analytical Vernier Effect for Silicon Panda Ring Resonator, in National Science Postgraduate Conference, NSPC 2011: Universiti Teknologi Malaysia.

[4] I.S. Amiri, K. Raman, A. Afroozeh, M. Jalil, I. Nawi, J. Ali, and P. Yupapin, Generation of DSA for Security Application, Procedia Engineering, 8, 2011, 360-365.

[5] N. Suwanpayak, S. Songmuang, M. Jalil, I.S. Amiri, I. Naim, J. Ali, and P. Yupapin. Tunable and storage potential wells using microring resonator system for bio-cell trapping and delivery. Enabling Science and Nanotechnology (ESciNano) Conference. 2010: IEEE Explore.

[6] P. Yupapin, M. Jalil, I.S. Amiri, I. Naim, and J. Ali, New Communication Bands Generated by Using a Soliton Pulse within a Resonator System, Circuits and Systems, 1, 2010.

[7] A. Afroozeh, M. Bahadoran, I.S. Amiri, A.R. Samavati, J. Ali, and P.P. Yupapin, Fast Light Generation Using GaAlAs/GaAs Waveguide, Jurnal Teknologi, 57, 2012, 7.

[8] A. Afroozeh, I.S. Amiri, M.A. Jalil, M. Kouhnavard, J. Ali, and P.P. Yupapin, Multi Soliton Generation for Enhance Optical Communication, Applied Mechanics and Materials, 83, 2011, 136-140.

[9] I. Amiri, A. Afroozeh, and M. Bahadoran, Simulation and Analysis of Multisoliton Generation Using a PANDA Ring Resonator System, Chinese Physics Letters, 28(10), 2011, 104205.

[10] I.S. Amiri, J. Ali, and P. Yupapin, Enhancement of FSR and Finesse Using Add/Drop Filter and PANDA Ring Resonator Systems, International Journal of Modern Physics B, 26(04), 2012.

[11] I.S. Amiri, M. Ranjbar, A. Nikoukar, A. Shahidinejad, J. Ali, and P. Yupapin. Multi optical Soliton generated by PANDA ring resonator for secure network communication. Computer and Communication Engineering (ICCCE) Conference 2012: IEEE Explore.

[12] I.S. Amiri, G. Vahedi, A. Nikoukar, A. Shojaei, J. Ali, and P. Yupapin, Decimal Convertor Application for Optical Wireless Communication by Generating of Dark and Bright Signals of soliton, International Journal of Engineering Research and Technology, 1(5), 2012.

[13] M.A. Jalil, I.S. Amiri, C. Teeka, J. Ali, and P. Yupapin, All-optical Logic XOR/XNOR Gate Operation using Microring and Nanoring Resonators, Global Journal of Physics Express, 1(1), 2011, 15-22.

[14] C. Tanaram, C. Teeka, R. Jomtarak, P. Yupapin, M. Jalil, I.S. Amiri, and J. Ali, ASK-to-PSK Generation based on Nonlinear Microring Resonators Coupled to One MZI Arm, Procedia Engineering, 8, 2011, 432-435.

[15] I.S. Amiri, M.A. Jalil, A. Afroozeh, M. Kouhnavard, J. Ali, and P.P. Yupapin, Controlling Center Wavelength and Free Spectrum Range by MRR Radii, in Faculty of Science Postgraduate Conference (FSPGC). 2010: UNIVERSITI TEKNOLOGI MALAYSIA.

[16] A. Afroozeh, I.S. Amiri, M. Kouhnavard, M. Bahadoran, M.A. Jalil, J. Ali, and P.P. Yupapin, Dark and Bright Soliton trapping using NMRR, in The International Conference on Experimental Mechanics (ICEM2010). 2010: Kuala Lumpur, Malaysia.

[17] A. Afroozeh, I.S. Amiri, J. Ali, and P. Yupapin, Determination Of FWHM For Soliton Trapping, Jurnal Teknologi, 55, 2012, 7783.

[18] A. Afroozeh, I.S. Amiri, M. Bahadoran, J. Ali, and P. Yupapin, Simulation Of Soliton Amplification In Micro Ring Resonator For Optical Communication, Jurnal Teknologi, 55, 2012, 271-277.

[19] A. Afroozeh, I.S. Amiri, A. Samavati, J. Ali, and P. Yupapin. THz frequency generation using MRRs for THz imaging. Enabling Science and Nanotechnology (ESciNano) Conference 2012: IEEE Explore.

[20] I.S. Amiri, A. Afroozeh, J. Ali, and P. Yupapin, Generation Of Quantum Codes Using Up And Down Link Optical Solition, Jurnal Teknologi, 55, 2012, 97-106.

[21] M. Hamdi, T. Saktioto, I.S. Amiri, and A. J., Transition of Diatomic Molecular Oscillator Process in THz Region, in The International Conference on Experimental Mechanics (ICEM) 2010 Kuala Lumpur, Malaysia.

[22] I.S. Amiri, A. Afroozeh, M. Bahadoran, J. Ali, and P. Yupapin, Molecular Transporter System For Qubits Generation, Jurnal Teknologi, 55, 2012, 155-165.

[23] I.S. Amiri, A. Nikoukar, and J. Ali, Quantum Information Generation Using Optical Potential Well, in Network Technologies \& Communications Conference. 2010-2011: Singapore.

[24] I.S. Amiri, A. Nikoukar, A. Shahidinejad, J. Ali, and P. Yupapin. Generation of discrete frequency and wavelength for secured computer networks system using integrated ring resonators. Computer and Communication Engineering (ICCCE) Conference 2012: IEEE Explore.

[25] I.S. Amiri, A. Nikoukar, G. Vahedi, A. Shojaei, J. Ali, and P. Yupapin, Frequency-Wavelength Trapping by Integrated Ring Resonators For Secured Network and Communication Systems, International Journal of Engineering Research \& Technology (IJERT), 1(5), 2012.

[26] M. Imran, R.A. Rahman, and I.S. Amiri, Fabrication of Diffractive Optical Element using Direct Writing CO2 Laser Irradiation, in Faculty of Science Postgraduate Conference (FSPGC). 2010 UNIVERSITI TEKNOLOGI MALAYSIA.

[27] I.S. Amiri, A. Shahidinejad, A. Nikoukar, J. Ali, and P. Yupapin, A Study oF Dynamic Optical Tweezers Generation For Communication Networks, International Journal of Advances in Engineering \& Technology (IJAET), 4(2), 2012, 38-45

[28] I.S. Amiri, G. Vahedi, A. Shojaei, A. Nikoukar, J. Ali, and P. Yupapin, Secured Transportation of Quantum Codes Using Integrated PANDA-Add/drop and TDMA Systems, International Journal of Engineering Research \& Technology (IJERT), 1(5), 2012.

[29] I.S. Amiri, A. Shahidinejad, A. Nikoukar, M. Ranjbar, J. Ali, and P.P. Yupapin, Digital Binary Codes Transmission via TDMA Networks Communication System Using Dark and Bright Optical Soliton, GSTF Journal on Computing (joc) 2 (1), 2012.

[30] I.S. Amiri, A. Nikoukar, A. Shahidinejad, M. Ranjbar, J. Ali, and P.P. Yupapin, Generation of Quantum Photon Information Using Extremely Narrow Optical Tweezers for Computer Network Communication, GSTF Journal on Computing (joc) 2 (1), 2012.

[31] M. Kouhnavard, I.S. Amiri, M. Jalil, A. Afroozeh, J. Ali, and P.P. Yupapin. QKD via a quantum wavelength router using spatial soliton. Enabling Science and Nanotechnology (ESciNano) Conference 2010: IEEE Explore.

[32] A. Nikoukar, I.S. Amiri, and J. Ali, Secured Binary Codes Generation for Computer Network Communication, in Network Technologies \& Communications (NTC) Conference. 2010-2011: Singapore.

[33] A. Afroozeh, M. Bahadoran, I.S. Amiri, A.R. Samavati, J. Ali, and P.P. Yupapin, Fast Light Generation Using Microring Resonators for Optical Communication, in National Science Postgraduate Conference NSPC. 2011: Universiti Teknologi Malaysia. 
[34] A. Afroozeh, I.S. Amiri, M. Kouhnavard, M. Bahadoran, M.A. Jalil, J. Ali, and P.P. Yupapin, Optical Memory Time using Multi Bright Soliton, in The International Conference on Experimental Mechanics 2010 (ICEM 2010) 2010: Kuala Lumpur, Malaysia.

[35] C. Teeka, S. Songmuang, R. Jomtarak, P. Yupapin, M. Jalil, I.S. Amiri, and J. Ali. ASK-to-PSK Generation based on Nonlinear Microring Resonators Coupled to One MZI Arm. International Conference On Enabling Science And Nanotechnology Escinano 2011. Kuala Lumpur, (Malaysia) AIP Conference Proceedings.

[36] I.S. Amiri, M.H. Khanmirzaei, M. Kouhnavard, P.P. Yupapin, and J. Ali, Quantum Entanglement using Multi Dark Soliton Correlation for Multivariable Quantum Router. Quantum Entanglement ed. A.M. Moran. 2012, New York: Nova Publisher.

[37] A. Nikoukar, I.S. Amiri, A. Shahidinejad, A. Shojaei, J. Ali, and P. Yupapin. MRR quantum dense coding for optical wireless communication system using decimal convertor. Computer and Communication Engineering (ICCCE) Conference. 2012: IEEE Explore.

[38] A. Shahidinejad, A. Nikoukar, I.S. Amiri, M. Ranjbar, A. Shojaei, J. Ali, and P. Yupapin. Network system engineering by controlling the chaotic signals using silicon micro ring resonator. Computer and Communication Engineering (ICCCE) Conference. 2012: IEEE Explore.

[39] M.A. Jalil, I.S. Amiri, M. Kouhnavard, A. Afroozeh, J. Ali, and P.P. Yupapin, Finesse Improvements of Light Pulses within MRR System, in Faculty of Science Postgraduate Conference (FSPGC). 2010: UNIVERSITI TEKNOLOGI MALAYSIA.

[40] M. Kouhnavard, A. Afroozeh, M.A. Jalil, I.S. Amiri, J. Ali, and P.P. Yupapin, Soliton Signals and the Effect of Coupling Coefficient in MRR Systems, in Faculty of Science Postgraduate Conference (FSPGC). 2010 UNIVERSITI TEKNOLOGI MALAYSIA.

[41] A.A. Shojaei and I.S. Amiri, Soliton for Radio wave generation, in The International Conference for Nanomaterials Synthesis and Characterization (INSC) Conference. 2011: kuala lumpur, MALAYSIA.

[42] A. Afroozeh, M. Kouhnavard, I.S. Amiri, M.A. Jalil, J. Ali, and P.P. Yupapin, Effect of Center Wavelength on MRR Performance, in Faculty of Science Postgraduate Conference (FSPGC). 2010: UNIVERSITI TEKNOLOGI MALAYSIA.

[43] A.A. Shojaei and I.S. Amiri, DSA for Secured Optical Communication, in The International Conference for Nanomaterials Synthesis and Characterization (INSC) Conference. 2011: kuala lumpur MALAYSIA.

[44] I.S. Amiri, A. Afroozeh, M. Bahadoran, J. Ali, and P.P. Yupapin, Up and Down Link of Soliton for Network Communication, in National Science Postgraduate Conference, NSPC. 2011. 